Canadian

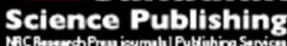

Applied Physiology, Nutrition, and Metabolism Physiologie appliquée, nutrition et métabolisme

\title{
Differential Impact of Visual Feedback on Plantar- and Dorsi-flexion Maximal Torque Output
}

\begin{tabular}{|r|l|}
\hline Journal: & Applied Physiology, Nutrition, and Metabolism \\
\hline Manuscript ID & apnm-2015-0639.R2 \\
\hline Manuscript Type: & Brief communication \\
\hline Date Submitted by the Author: & 23-Jan-2016 \\
\hline Complete List of Authors: & $\begin{array}{l}\text { Toumi, Anis; Université de Valenciennes et du Hainaut Cambrésis, LAMIH } \\
\text { UMR CNRS 8201 Campus du Mont Houy Valenciennes, FR 59313 } \\
\text { Jakobi, Jennifer; University of British Columbia, Health and Exercise } \\
\text { Sciences } \\
\text { SIMONEAU-BUESSINGER, Emilie; Université de Valenciennes et du Hainaut } \\
\text { Cambrésis, LAMIH UMR CNRS 8201 }\end{array}$ \\
\hline Keyword: & $\begin{array}{l}\text { Ankle joint, Force, Isometric, Maximal voluntary contractions, Motivation, } \\
\text { Muscle activation, Neuromuscular performance,Strength }\end{array}$ \\
\hline
\end{tabular}

\section{SCHOLARONE}

Manuscripts 


\section{Differential Impact of Visual Feedback on Plantar- and Dorsi-flexion Maximal Torque Output}

Anis TOUMI ${ }^{1}$, Jennifer M. JAKOBI ${ }^{2}$, Emilie SIMONEAU-BUESSINGER ${ }^{1^{*}}$

${ }^{1}$ Laboratoire d'Automatique, de Mécanique, et d'Informatique industrielles et Humaines (LAMIH) - UMR CNRS 8201, Université de Valenciennes et du Hainaut-Cambrésis (UVHC), F59313 Valenciennes, France

${ }^{2}$ School of Health and Exercise Sciences, University of British Columbia Okanagan, Kelowna, Canada

*Corresponding author: Emilie Simoneau-Buessinger, Laboratoire d'Automatique, de Mécanique, et d'Informatique industrielles et Humaines (LAMIH) - UMR CNRS 8201, Université de Valenciennes et du Hainaut-Cambrésis (UVHC), Le Mont Houy, F-59313 Valenciennes Cedex 09, France; Tel : + 33327511460 E-mail: emilie.simoneau@univ-valenciennes.fr

Running Title: Visual feedback influence on muscular performance 


\begin{abstract}
The effect of visual feedback on enhancing isometric maximal voluntary contractions (MVC) was evaluated. Twelve adults performed plantar-flexion and dorsi-flexion MVCs in three conditions (no visual feedback, visual feedback, visual feedback with target). There was no significant effect of visual conditions on dorsi-flexion MVC but there was an effect on plantarflexion. Irrespective of whether a target was evident, visual feedback increased plantar-flexion MVC $\sim 15 \%$. This study highlights the importance of optimal feedback to enhance MVC.
\end{abstract}

\title{
KEY WORDS
}

Ankle joint, Force enhancement, Isometric, Lower limb, Maximal voluntary contractions, Muscle activation, Neuromuscular performance, Strength, Target 


\section{INTRODUCTION}

Isometric maximal voluntary contraction (MVC) is commonly used to evaluate muscle plasticity across acute and chronic interventions, for example fatigue or training. As well, an MVC is regularly applied for the normalization of submaximal loads between individuals differing in strength, such as young and old or males and females. A variety of factors influence MVC torque including time of day (Martin et al. 1999), practice (familiarization) (Graves et al. 1990), temperature (Morrison et al. 2004), ventilation (Li and Laskin 2006), prior physical or mental activities (Bishop 2003, Marcora et al. 2009), dynamometer (Toumi et al. 2015) and instructions given (Christ et al. 1993). To quantify whether factors such as these, or physiological differences between individuals influence the production of an MVC, the twitch interpolation technique and measures of the central activation ratio are often employed (Klass 2005, Simoneau et al. 2006). Although utilization of these electrical stimulation methods are ideal to ensure assessment of maximal torque production, they are not always feasible in workplace, clinical and exercise settings or across all persons and conditions, thus the assessment of MVC is often necessary without the application of these quantifiable techniques.

In many instances, an MVC can be modulated by the level of motivation and feedback offered (Gandevia 2001). There is a positive relationship between the volume of verbal commands and the strength of the voluntary motor response to the extent that a $5 \%$ increase in peak elbow flexion MVC was evident when verbal encouragement was offered (Johansson et al. 1983, McNair et al. 1996) and when visual feedback was combined with verbal encouragement, rate of torque development was faster (Campenella et al. 2000). Visual feedback is a well-known moderator of force variability (Christou 2005), rehabilitation progress (van Vliet and Wulf 2015), balance (Sayenko et al. 2010) and joint coordination (Freitas and Duarte 2012). However, to-date 
no study has evaluated the use of a target torque with real-time visual feedback for the purpose of increasing maximal torque.

Plantar-flexion (PF) and dorsi-flexion (DF) torques are widely used in activities of daily living, such as standing balance, locomotion or vehicular driving. As a consequence, researchers and clinicians often focus on the ankle joint muscles and an accurate assessment of PF and DF MVC torques is then particularly necessary. The aim of this study was thus to assess the effect of using a target coupled with real-time visual feedback of torque on isometric PF and DF MVC torques. One could assume that the provision of visual feedback coupled with a target line would enhance maximal torque.

\section{MATERIALS AND METHODS}

\section{Participants}

Twelve healthy adults (6 males and 6 females) (age: $24.0 \pm 2.3$ years, body mass: $63.2 \pm 8.9 \mathrm{~kg}$, height: $1.67 \pm 0.07 \mathrm{~m}$ ) participated. All participants were previously familiarized with the experimental setup and all self-identified as right leg dominant. Written consent was obtained and all procedures complied with the Declaration of Helsinki and were approved by the local ethics committee. At the time of testing, participants had no history of recent musculoskeletal injury in the examined leg as well as no visual problems.

\section{Experimental setup}

An ankle ergometer 'Booted, Open-unit device, Three dimension sensor, Transportable, Ergometer (B.O.T.T.E.)' was used to record isometric torque at the ankle joint in both DF and PF (Toumi et al. 2015). The participant was seated with the hip at $\sim 60^{\circ}$, the dominant leg extended straight forward and the ankle joint placed at $0^{\circ}$ (the angle between the plantar surface and the leg 
was a right angle). An orthosis was fastened from approximately mid-shank to mid-thigh on the leg to ensure it was extended forward for the duration of the experiment. The participant's arms were folded across the chest during testing. The axis of rotation of the ankle joint was aligned with the axis of rotation of the ergometer. Special care was taken to prevent any movement of the lower extremity during isometric DF and PF contractions. Torque was sampled at $100 \mathrm{~Hz}$ using a custom built Matlab program (The MathWorks Inc., USA) and a 16-bit A/D board (National Instruments, Texas, USA) interfaced to a personal computer.

\section{Experimental protocol}

Participants performed a standardized warm-up consisting of approximately 10 brief and nonfatiguing submaximal isometric contractions of increasing intensity (up to almost $80 \% \mathrm{MVC}$ ) in both DF and PF. This warm-up also allowed the participants to become accustomed to the test procedure. A computer screen was placed at eye level $1.5 \mathrm{~m}$ in front of the participant for realtime visual feedback of torque during the contractions as well as the target line. Half of the participants were first tested in PF then in DF, and vice versa for the other half. Three experimental screen conditions were tested for both DF and PF, in a randomized order: no visual feedback, visual feedback and visual feedback with target line (Figure 1). In the no visual feedback condition, at least two MVCs were executed with a blank screen in front of the participant. If the two MVCs were not within 5\% of each other additional trials were performed. The visual feedback condition was executed similarly to no feedback except a rectangle appeared on the screen and its height increased with the amount of torque output. In the visual feedback with target line condition, the real-time visual feedback display, inclusive of gain, was kept constant to the visual feedback condition but a horizontal target line was added on the screen, and positioned at 5\% above the previous MVC. When the visual feedback with target line occurred 
first in the randomized order, one MVC was executed to establish the target. Participants were then encouraged to exceed the given target line. The gain of the visual feedback was optimized to the PF and DF conditions and kept consistent across the visual feedback and visual feedback with target line conditions. For all conditions participants were asked to produce force as rapidly as possible and once maximal was achieved to hold the explosive contraction for $\sim 5$-s. During all MVC attempts, participants were given strong verbal encouragement. A resting time of at least 2min was taken between each trial.

\section{Data analysis}

The maximal torque corresponded to the highest output that occurred over a 0.5 -s period within the 5-s maximal effort. The highest maximal torque from each condition was used for further analysis. All data processing was performed during the experiment using a custom built Matlab program (The MathWorks Inc., USA).

\section{Statistical analysis}

Statistical tests were performed with Statistica ${ }^{\circledR}$ software (StatSoft, USA). Data are reported as means \pm standard deviations in the text and figure. Normality of the data was checked with Shapiro-Wilk's test and equality of variances verified by the Levene test. To assess the differences in MVC torques during DF and PF between the three testing conditions, a two-way repeated measures ANOVA for Action (DF, PF) x Screen condition (no visual feedback, visual feedback, visual feedback with target line) was performed. Tukey HSD post-hoc tests were performed to determine where significant differences occurred. For all analyses, the level of significance was set at $\mathrm{p}<0.05$. Partial eta-squared $\left(\eta_{\mathrm{p}}{ }^{2}\right)$ was calculated for statistically significant parameters to estimate effect size. 


\section{RESULTS}

There was a significant Action $\mathrm{x}$ Screen condition interaction $\left(\mathrm{F}_{2,22}=13.23, \mathrm{p}<0.001\right.$, $\left.\eta_{\mathrm{p}}{ }^{2}=0.55\right)$. While there was no significant effect of screen condition on DF MVC torque ( $p=0.99$ for each comparison), there was a significant effect in PF. The MVC PF torques with the visual feedback condition without the target line $(142.8 \pm 44.0$ N.m $)$ and with the target line $(149.7 \pm 47.9$ N.m) did not differ from each other $(\mathrm{p}=0.56)$ but were significantly higher than those without feedback $(119.5 \pm 42.5$ N.m) $(\mathrm{p}<0.001$ for both comparisons) (Figure 1$)$.

\section{DISCUSSION}

The provision of a motivational goal, offered as a target line, did not provide added benefit in the production of maximal torque. Moreover, visual feedback of torque did not influence DF MVC; however, PF MVC torque increased more than $15 \%$ with visual feedback compared to without. These disparate outcomes between maximal DF and PF MVC underscore the importance of widely applying visual feedback, as its impact is muscle dependent. The assumption of an effect of visual feedback on performance will safeguard in the execution, evaluation and comparison of outcomes between studies when MVC is the measure of maximal performance or applied for the purpose of normalization.

When visual feedback is not used, and most notably in the PF, an underestimation of torque of at least $15 \%$ might occur. A diminution in MVC torque would reduce estimates of submaximal targets and over approximate maximal torque impacting numerous outcome measures. For example, underestimating maximal torque prior to a training program would likely culminate in 
observations of higher strength gains in exercise interventions. Although the effect of visual feedback on enhancing an MVC is poorly reported, the positive effect of visual feedback on the acquisition of finite motor skills (Horeman et al. 2012), postural control (Boudrahem and Rougier 2009) and reducing force fluctuations (Christou 2005) is well known. Thus, the benefit of visual feedback on the execution of MVC is unsurprising; however, its differential effect between agonist-antagonist pairs requires further study.

Since PF and DF torques are widely used in activities of daily living, such as standing balance, locomotion or vehicular driving, clinicians and researchers often focus on the ankle joint muscles, thus an accurate assessment of PF and DF MVC torques is necessary. Sources of variation that may influence muscle function and the production of MVC must be minimized in order to maximize precision in assessments of maximal performance. This experiment highlights that visual feedback influences maximal performance, therefore its provision is critical in the assessment of MVC; however, the effect does not apply equally across all muscle groups. The differential effect of visual feedback between DF and PF might surround the ability to maximally activate these muscles. Maximal voluntary activation is typically achieved for DF, but low, or 'incomplete' voluntary activation is repeatedly evident in the literature for PF (Belanger and McComas 1981, Simoneau et al. 2005). The ability to produce an MVC is influenced by numerous factors arising from descending drive generated in the motor cortex through to the recruitment and optimal discharge of motor units that is shaped through synaptic efficiency of excitatory and inhibitory feedback to the motoneuron pool (Taylor 2009). Thus, there are numerous sites for differences in voluntary activation to arise and be adjusted between muscles. 
Many experimental approaches rely on measurements of MVC, thus understanding factors that influence force generation is imperative in research design. Visual feedback influences maximal torque output and can be used as a source of information to encourage maximum torque production. The potential to influence maximal performance through visual feedback is as important for scientists in executing experiments as it is for coaches and clinicians for skill development and rehabilitation. It seems that a target or goal that offers real-time feedback of personal performance is a key factor in producing a 'true' MVC. By using visual feedback the researcher or therapist might be able to improve the accuracy and consistency of examination, and increase the efficacy and efficiency of athletic performance and clinical interventions through optimizing experimental design for achieving maximal force.

\section{CONFLICT OF INTEREST}

The authors declare that there are no conflicts of interest. 


\section{REFERENCES}

Allin, S., Matsuoka, Y., and Klatzky, R. 2002. Measuring just noticeable differences for haptic force feedback: implications for rehabilitation. In Proceedings 10th Symposium on Haptic Interfaces for Virtual Environment and Teleoperator Systems. HAPTICS 2002. IEEE Comput. Soc. pp. 299-302. doi: 10.1109/HAPTIC.2002.998972.

Belanger, A.Y., and McComas, A.J. 1981. Extent of motor unit activation during effort. J. Appl. Physiol. 51(5): 1131-5.

Bishop, D. 2003. Warm up II: performance changes following active warm up and how to structure the warm up. Sports Med. 33(7): 483-98.

Boudrahem, S., and Rougier, P.R. 2009. Relation between postural control assessment with eyes open and centre of pressure visual feedback effects in healthy individuals. Exp. Brain Res. 195(1): 145-152. doi: 10.1007/s00221-009-1761-1.

Campenella, B., Mattacola, C.G., and Kimura, I.F. 2000. Effect of visual feedback and verbal encouragement on concentric quadriceps and hamstrings peak torque of males and females. Isokinet. Exerc. Sci. 8: 1-6.

Christ, C.B., Boileau, R.A., Slaughter, M.H., Stillman, R.J., and Cameron, J. 1993. The effect of test protocol instructions on the measurement of muscle function in adult women. J. Orthop. Sports Phys. Ther. 18(3): 502-10. JOSPT, Inc. JOSPT, 1033 North Fairfax Street, Suite 304, Alexandria, VA 22134-1540. doi: 10.2519/jospt.1993.18.3.502.

Christou, E.A. 2005. Visual feedback attenuates force fluctuations induced by a stressor. Med. Sci. Sports Exerc. 37(12): 2126-33. 
Freitas, S.M.S.F., and Duarte, M. 2012. Joint coordination in young and older adults during quiet stance: Effect of visual feedback of the center of pressure. Gait Posture 35(1): 83-87. doi: 10.1016/j.gaitpost.2011.08.011.

Gandevia, S.C. 2001. Spinal and supraspinal factors in human muscle fatigue. Physiol. Rev. 81(4): 1725-89.

Graves, J.E., Pollock, M.L., Carpenter, D.M., Leggett, S.H., Jones, A., MacMillan, M., and Fulton, M. 1990. Quantitative assessment of full range-of-motion isometric lumbar extension strength. Spine (Phila. Pa. 1976). 15(4): 289-94.

Horeman, T., Rodrigues, S.P., van den Dobbelsteen, J.J., Jansen, F.-W., and Dankelman, J. 2012. Visual force feedback in laparoscopic training. Surg. Endosc. 26(1): 242-8. doi: 10.1007/s00464-011-1861-4.

Johansson, C.A., Kent, B.E., and Shepard, K.F. 1983. Relationship between verbal command volume and magnitude of muscle contraction. Phys. Ther. 63(8): 1260-5.

Klass, M. 2005. Aging does not affect voluntary activation of the ankle dorsiflexors during isometric, concentric, and eccentric contractions. J. Appl. Physiol. 99(1): 31-38. doi: 10.1152/japplphysiol.01426.2004.

Li, S., and Laskin, J.J. 2006. Influences of ventilation on maximal isometric force of the finger flexors. Muscle Nerve 34(5): 651-5. doi: 10.1002/mus.20592.

Marcora, S.M., Staiano, W., and Manning, V. 2009. Mental fatigue impairs physical performance in humans. J. Appl. Physiol. 106(3): 857-64. doi: 10.1152/japplphysiol.91324.2008.

Martin, A., Carpentier, A., Guissard, N., van Hoecke, J., and Duchateau, J. 1999. Effect of time of day on force variation in a human muscle. Muscle Nerve 22(10): 1380-7. 
McNair, P.J., Depledge, J., Brettkelly, M., and Stanley, S.N. 1996. Verbal encouragement: effects on maximum effort voluntary muscle action. Br. J. Sports Med. 30(3): 243-5.

Morrison, S., Sleivert, G.G., and Cheung, S.S. 2004. Passive hyperthermia reduces voluntary activation and isometric force production. Eur. J. Appl. Physiol. 91(5-6): 729-36. doi: 10.1007/s00421-004-1063-z.

Sayenko, D.G., Alekhina, M.I., Masani, K., Vette, A.H., Obata, H., Popovic, M.R., and Nakazawa, K. 2010. Positive effect of balance training with visual feedback on standing balance abilities in people with incomplete spinal cord injury. Spinal Cord 48(12): 886-93. doi: $10.1038 /$ sc.2010.41.

Simoneau, E., Martin, A., and Van Hoecke, J. 2005. Muscular performances at the ankle joint in young and elderly men. J. Gerontol. A. Biol. Sci. Med. Sci. 60(4): 439-47.

Simoneau, E., Martin, A., Porter, M.M., and Van Hoecke, J. 2006. Strength training in old age: adaptation of antagonist muscles at the ankle joint. Muscle Nerve 33(4): 546-55. doi: 10.1002/mus.20492.

Taylor, J.L. 2009. Point: the interpolated twitch does/does not provide a valid measure of the voluntary activation of muscle. J. Appl. Physiol. 107(1): 354-5. doi: 10.1152/japplphysiol.91220.2008.

Toumi, A., Leteneur, S., Gillet, C., Debril, J.-F., Decoufour, N., Barbier, F., Jakobi, J.M., and Simoneau-Buessinger, E. 2015. Enhanced precision of ankle torque measure with an openunit dynamometer mounted with a 3D force-torque sensor. Eur. J. Appl. Physiol. doi: 10.1007/s00421-015-3210-0.

van Vliet, P.M., and Wulf, G. 2015. Extrinsic feedback for motor learning after stroke: What is 
the evidence? Disabil. Rehabil. 28(13-14): 831-840. Taylor \& Francis. doi: $10.1080 / 09638280500534937$. 


\section{FIGURE CAPTIONS}

Figure 1. Dorsi-flexion and plantar-flexion maximal voluntary isometric torques depending on the provision of a real-time visual feedback with and without a target line.

The gain of the visual feedback was optimized for the maximal voluntary contraction and kept consistent across feedback conditions for the plantar-flexion as well as dorsi-flexion contractions.

* Significant difference between values $(\mathrm{p}<0.001)$ 

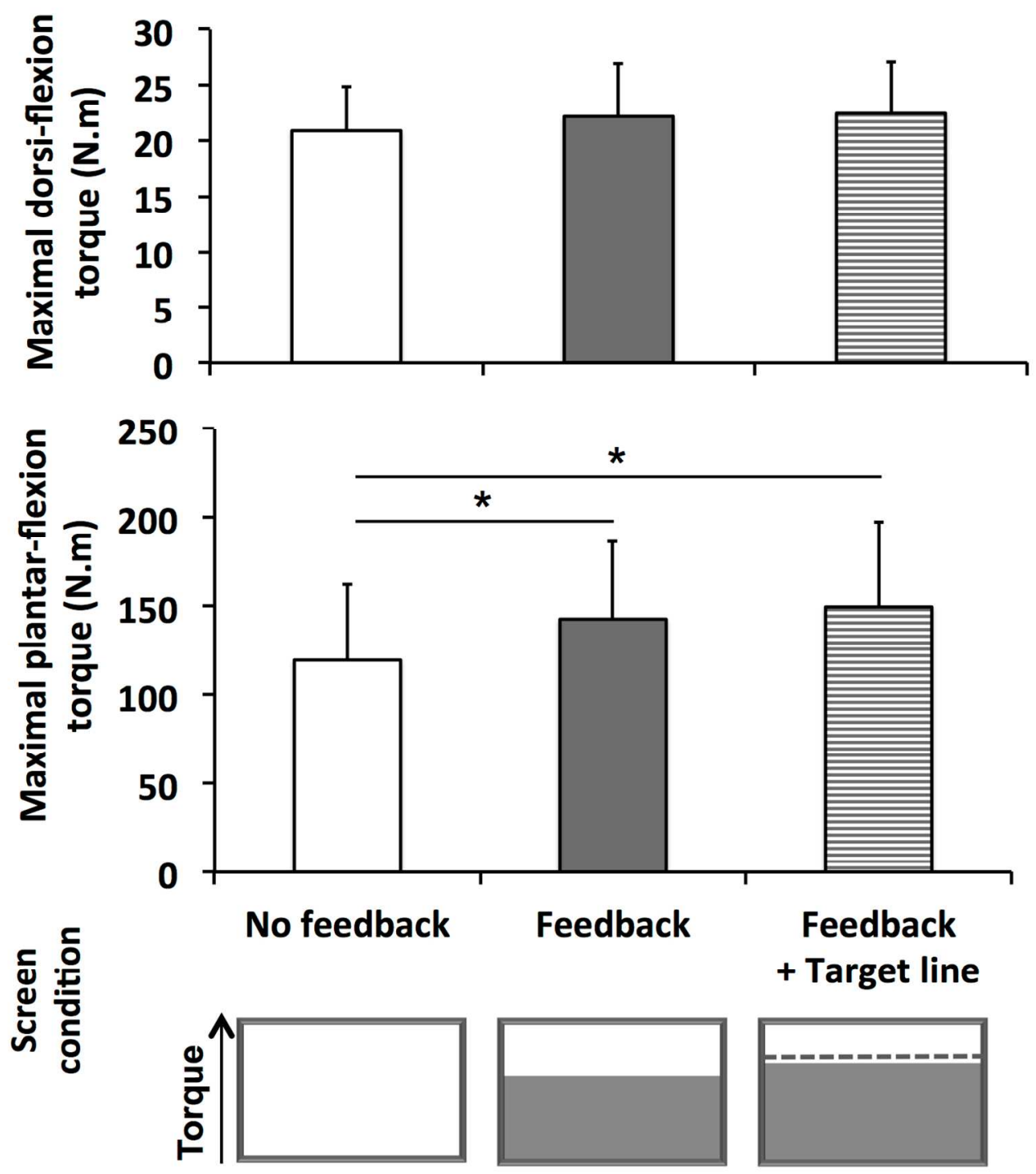

Dorsi-flexion and plantar-flexion maximal voluntary isometric torques depending on the provision of a realtime visual feedback with and without a target line. The gain of the visual feedback was optimized for the maximal voluntary contraction and kept consistent across feedback conditions for the plantar-flexion as well as dorsi-flexion contractions. * Significant difference between values $(p<0.001)$. 